\title{
Melatonin Protects Chronic Kidney Disease Mesenchymal Stem/Stromal Cells against Accumulation of Methylglyoxal via Modulation of Hexokinase-2 Expression
}

\author{
Gyeongyun Go ${ }^{1,2}$, Yeo Min Yoon ${ }^{3}$, Sungtae Yoon ${ }^{4}$, Gaeun Lee ${ }^{1,2}$, Ji Ho Lim ${ }^{1}$, Su-Yeon Han $^{4}$ and \\ Sang Hun Lee L,2,3,4,* $^{1,2}$ \\ ${ }^{1}$ Department of Biochemistry, Soonchunhyang University College of Medicine, Cheonan 31151, \\ 2Department of Biochemistry, BK21FOUR Project2, College of Medicine, Soonchunhyang University, Cheonan 31151, \\ ${ }^{3}$ Medical Science Research Institute, Soonchunhyang University Seoul Hospital, Seoul 04401, \\ ${ }^{4}$ Stembio Ltd., Asan 31538, Republic of Korea
}

\begin{abstract}
Treatment options for patients with chronic kidney disease (CKD) are currently limited; therefore, there has been significant interest in applying mesenchymal stem/stromal cell (MSC)-based therapy to treat CKD. However, MSCs harvested from CKD patients tend to show diminished viability and proliferation due to sustained exposure to uremic toxins in the CKD environment, which limits their utility for cell therapy. The application of melatonin has been demonstrated to improve the therapeutic efficacy of MSCs derived from and engrafted to tissues in patients suffering from CKD, although the underlying biological mechanism has not been elucidated. In this study, we observed overexpression of hexokinase-2 (HK2) in serum samples of CKD patients and MSCs harvested from an adenine-fed CKD mouse model (CKD-mMSCs). HK2 upregulation led to increased production levels of methylglyoxal (MG), a toxic metabolic intermediate of abnormal glycolytic processes. The overabundance of HK2 and MG was associated with impaired mitochondrial function and low cell proliferation in CKD-mMSCs. Melatonin treatment inhibited the increases in HK2 and MG levels, and further improved mitochondrial function, glycolytic metabolism, and cell proliferation. Our findings suggest that identifying and characterizing metabolic regulators such as HK2 in CKD may improve the efficacy of MSCs for treating CKD and other kidney disorders.
\end{abstract}

Key Words: Melatonin, Hexokinase, Methylglyoxal, Mesenchymal stem/stromal cells, Mitochondria, Glycolysis

\section{INTRODUCTION}

Chronic kidney disease (CKD) remains a highly prevalent condition that affects all ages and has a high mortality rate (Lv and Zhang, 2019), constituting a significant portion of the global disease burden (Romagnani et al., 2017). Despite the great efforts made by both clinical and basic science researchers to develop an effective therapy, the burden of CKD has not declined over the past few decades (GBD Chronic Kidney Disease Collaboration, 2020). Progression of kidney injury in CKD leads to ultimate loss of kidney function, which causes the accumulation of uremic toxins in the kidney tissues (Nigam and Bush, 2019), significantly increasing the mortality and risk of morbidity (Lee et al., 2018). Mitochondrial damage and dysfunction play a critical role in the pathophysiology of kidney injury. Despite ongoing efforts to elucidate the specific regulatory mechanisms of mitochondrial dysregulation in CKD, currently available knowledge is insufficient to design clinically successful interventions without adverse side effects (Breyer and Susztak, 2016).

Mesenchymal stem/stromal cells (MSCs) are attractive candidates for developing cell-based therapies for CKD, as they can contribute to tissue repair or regeneration by releasing appropriate factors related to cell proliferation, survival, and differentiation (Yun and Lee, 2019). Several reports have suggested that the angiogenic and regenerative properties of MSCs can improve various pathological conditions in vivo $\mathrm{Hu}$ and Li, 2018; Keshtkar et al., 2018; Thalakiriyawa et al., 2021), supporting the idea that MSC-based therapy may be highly efficacious for treating CKD and other renal diseases. However,

\section{Open Access https://doi.org/10.4062/biomolther.2021.058}

This is an Open Access article distributed under the terms of the Creative Commons Attribution Non-Commercial License (http://creativecommons.org/licenses/by-nc/4.0/) which permits unrestricted non-commercial use, distribution, and reproduction in any medium, provided the original work is properly cited.
Received Mar 29, 2021 Revised May 8, 2021 Accepted Jun 3, 2021

Published Online Jul 15, 2021

* Corresponding Author

E-mail: shlee0551@gmail.com

Tel: +82-2-709-9029, Fax: +82-2-792-5812 
CKD patient-derived human MSCs (CKD-hMSCs) show decreased potency for autologous cell-based therapy because of their exposure to uremic toxins circulating in the body during their growth and after their administration to CKD patients (Hickson et al., 2016). Therefore, more work is needed to find an improved method for increasing and protecting MSCs in the context of CKD to realize their therapeutic potential.

Melatonin is a peptide hormone released by the pineal gland to modulate several physiological functions, including sleep, but there has been growing interest in its broader therapeutic potential. Accumulating evidence suggests that melatonin not only ameliorates myocardial infarction (Fu et al., 2020), pulmonary ischemia reperfusion injury (Bhatia et al., 2019), and kidney injury (Ohashi et al., 2019) when administered directly but also improves the viability, motility, and proliferation of MSCs (Hu and Li, 2019), thereby enhancing the therapeutic potential of MSC-based therapy (Yoon et al., 2020c). Our previous work demonstrated that melatonin treatment can improve the stress tolerance of mouse MSCs (mMSCs) by improving mitochondrial function (Han et al., 2019). In a different study, we found that prion protein-rich exosomes from melatonin-treated normal hMSCs can modulate microRNA-4516 in CKD-hMSCs to improve their therapeutic potential (Yoon et al., 2020c). Although melatonin-induced improvements in mitochondrial ATP synthesis have been confirmed in vitro and in vivo, the mechanism by which melatonin engages the broader metabolic network in the context of its protective effect against CKD has not been well investigated.

Hexokinase-2 (HK2) catalyzes the ATP-mediated phosphorylation of glucose to glucose-6-phosphate (G6P), which is the rate-limiting step of glucose metabolism. Overexpression of HK2 has been linked to diabetic nephropathy (Rabbani and Thornalley, 2019), or diabetic kidney disease, which is another form of CKD induced by hyperglycemia (Kato and Natarajan, 2019). Elevation of HK2 has also been speculated to be related to ischemic CKD (Rabbani and Thornalley, 2019). Although HK2 isoforms have distinct roles in non-pathologic cellular processes, HK2 overexpression can occur in some pathogenic contexts such as in mediating tumorigenic activity (Liu et al., 2017 ) or the profibrotic actions of transforming growth factor- $\beta$ (Yin et al., 2019). A recent report suggested that HK2 is usually bound to the voltage-dependent anion channel (VDAC) on the outer mitochondrial membrane, but abnormally high cytosolic glucose concentrations displace and stabilize HK2 without upregulating other enzymes involved in glycolysis. This causes the accumulation of methylglyoxal (MG) and MGderived advanced glycation end-products, all of which contribute to dysregulation of metabolism, leading to mitochondrial dysfunction and oxidative stress.

In the current study, we investigated whether HK2 overexpression is related to non-diabetic CKD and MSCs from the CKD condition. Our data showed that HK2 expression was markedly elevated in the serum of CKD patients, as well as in MSCs derived from a CKD mouse model (CKD-mMSCs). Thus, we hypothesized that melatonin may influence the accumulation of abnormal glycolytic intermediates, or glycolytic overload, to improve mitochondrial function and protect MSCs against CKD. Melatonin-treated CKD-mMSCs demonstrated lower HK2 expression, a reduced MG concentration, improved mitochondrial function, and normal glycolysis. Collectively, our data show that melatonin downregulates HK2 and MG to improve the therapeutic potential of MSCs for CKD.

\section{MATERIALS AND METHODS}

\section{Serum collection of CKD patients and healthy controls}

The local ethics committee approved this study, and informed consent was obtained from all individuals participating in the study. Explanted sera $(n=30)$ were obtained from patients with CKD at Gyeongsang National University, Jinju, Korea (IRB: SCHUH 2018-04-035-002). Upon fulfilling the transplantation criteria, the control samples were obtained from healthy controls $(n=30)$ at the Korea Institute of Radiological \& Medical Sciences (IRB: SCHUH 2018-04-035-002). CKD diagnoses were made based on abnormal kidney function with an estimated glomerular filtration rate $<25 \mathrm{~mL} \cdot \mathrm{min}^{-1} \bullet 1.73 \mathrm{~m}^{-2}$ for over 3 months (stages 3b-5).

\section{Establishment of the CKD mouse model and harvesting bone marrow-derived MSCs}

We prepared a CKD mouse model following a previously established protocol (Yoon et al., 2020a, 2020b). In brief, 8-week-old male BALB/c mice were fed an adenine-containing diet $(0.75 \%$ adenine in diet) for $1-2$ weeks. Mouse body weights were measured weekly. Bone marrow-derived MSCs were harvested from the hind limb long bones. Isolated bone marrow was centrifuged and suspended in primary culture medium, and bone marrow-derived MSCs were cultured in a humidified incubator at $37^{\circ} \mathrm{C}$ with $5 \% \mathrm{CO}_{2}$. Healthy- and CKDMSCs were separated from each group with five mice per group. All MSCs were characterized in each cell line.

\section{Detection of MG in serum and cellular lysates}

The concentrations of $M G$ in the human healthy control group or CKD patient group, or in whole cell lysates of healthy mMSCs, CKD-mMSCs, and CKD-mMSCs treated with melatonin were determined using a commercially available enzyme-linked immunosorbent assay (ELISA) kit (Lifespan Biosciences, Seattle, WA, USA). A total of $100 \mu \mathrm{L}$ of the sample from each group was used for the experiments. The levels of MG were quantified by measuring the absorbance at $450 \mathrm{~nm}$ using a microplate reader (BMG Labtech, Ortenberg, Germany).

\section{Melatonin treatment to mMSCs}

Healthy- and CKD-mMSCs were washed twice with phosphate-buffered saline (PBS), and then cultured in fresh alpha-minimal essential medium (HyClone, Logan, UT, USA) supplemented with $10 \%$ fetal bovine serum (GIBCO, Grand Island, NY, USA). To examine the protective effect of melatonin (Sigma-Aldrich, St. Louis, MO, USA), mMSCs were incubated with melatonin $(1 \mu \mathrm{M})$ at $37^{\circ} \mathrm{C}$ for $24 \mathrm{~h}$ and then subjected to various experimental assays for respective purposes. For luzindole (Sigma-Aldrich) treatment, the mMSCs were pretreated with luzindole ( $1 \mu \mathrm{M}$ for $48 \mathrm{~h}$ ) before melatonin treatment.

\section{Preparation of the mitochondrial fraction}

The harvested healthy-mMSCs or CKD-mMSCs were lysed in mitochondria lysis buffer (Thermo Fisher Scientific, Waltham, MA, USA) and were subsequently incubated for 10 min on ice. The mixtures were centrifuged at $5,000 \mathrm{~g}$ at $4^{\circ} \mathrm{C}$ for $20 \mathrm{~min}$. The residual pellet containing the mitochondrial fraction was lysed with RIPA lysis buffer (Thermo Fisher Scientific) and then used for subsequent analysis. 


\section{Western blot analysis}

Whole cell lysates, cytosol fraction lysates, or mitochondrial lysates from healthy-mMSCs or CKD-mMSCs (30 $\mu$ g protein) were separated by sodium dodecyl sulfate-polyacrylamide gel electrophoresis on an $8 \%-12 \%$ gel, and the proteins were transferred to a nitrocellulose membrane. After the blots were washed with TBST (10 mM Tris- $\mathrm{HCl}$ [pH 7.6], $150 \mathrm{mM} \mathrm{NaCl}$, $0.05 \%$ Tween 20 ), the membranes were blocked with $5 \%$ skim milk for $1 \mathrm{~h}$ at room temperature and incubated with the appropriate primary antibodies against HK1 (\#2024S, Cell Signaling Technology, Beverly, MA, USA), HK2 (\#2867S, Cell Signaling Technology), VDAC1 (NB100-695, Novus), CDK2 (sc-6248, Santa Cruz Biotechnology, Santa Cruz, CA, USA), CDK4 (SC-56277, Santa Cruz Biotechnology), cyclin D1 (SC-20044, Santa Cruz Biotechnology), cyclin E (SC-377100, Santa Cruz Biotechnology), and $\beta$-actin (sc-47778, Santa Cruz Biotechnology). The membranes were then washed, and the primary antibodies were detected using goat anti-rabbit IgG or goat anti-mouse IgG secondary antibodies (Santa Cruz Biotechnology). The bands were detected by enhanced chemiluminescence (Amersham Pharmacia Biotech, Little Chalfont, UK). Densitometric quantification was performed using ImageJ software 1.48v (National Institutes of Health, Bethesda, MD, USA).

\section{Measurement of mitochondrial superoxide and mitochondrial membrane potential}

The production of mitochondrial superoxide in mMSCs was measured using MitoSOX (Thermo Fisher Scientific). To measure mitochondrial membrane potential, tetramethylrhodamine, methyl ester, and perchlorate (TMRE; Thermo Fisher Scientific) dye was used. The mMSCs in each group were trypsinized and centrifuged at 1,200 rpm for $3 \mathrm{~min}$. After washing with PBS twice, the cells were treated with either $10 \mu \mathrm{M}$ MitoSOX in PBS or $200 \mathrm{nM}$ TMRE in PBS at $37^{\circ} \mathrm{C}$ for $15 \mathrm{~min}$. MitoSOX-positive or TMRE-positive cells were analyzed using flow cytometry (Sysmex, Kobe, Japan). Flow cytometry data were analyzed using FCS Express 5 Flow research software (DeNovo Software, Los Angeles, CA, USA).

\section{Measurement of oxygen consumption rate (OCR) and extracellular acidification rate (ECAR) with a Seahorse assay}

OCR and ECAR were measured in mMSCs using an XF96 extracellular flux analyzer (Seahorse Bioscience, MA, USA). In brief, mMSCs were seeded at 45,000 cells per well in XF96 cell culture multi-well plates in Dulbecco's modified Eagle medium, and incubated for $24 \mathrm{~h}$ at $37^{\circ} \mathrm{C}$ and $5 \% \mathrm{CO}_{2}$. The XF96 cartridges were then incubated overnight in an XF calibrant at $37^{\circ} \mathrm{C}$ in a non- $\mathrm{CO}_{2}$ incubator. After the growth medium of mMSCs was changed with XF medium, the plates were incubated at $37^{\circ} \mathrm{C}$ in a non- $\mathrm{CO}_{2}$ incubator for $1 \mathrm{~h}$. Inhibitors were diluted to appropriate concentrations in XF medium and loaded into corresponding microwells in the XF96 cartridge plate. Following the equilibration of the sensor cartridges, the XF96 cell culture plate was loaded into the XF96 Extracellular Flux analyzer at $37^{\circ} \mathrm{C}$. OCR and ECAR were measured after cycles of mixing and data acquisition (basal), or inhibitor injection, mixing, and data acquisition using Seahorse Wave Desktop software (Agilent Technologies, Santa Clara, CA, USA).

\section{Ethics statement}

All animal care procedures and experiments were approved by the Institutional Animal Care and Use Committee of Soonchunhyang University Seoul Hospital (Seoul, Korea) (IACUC2013-5) and performed in accordance with the National Research Council (NRC) Guidelines for the Care and Use of Laboratory Animals. All animal experiments were performed using 8-week-old male BALB/c mice (Biogenomics, Seoul, Korea). The animals were maintained on a $12 \mathrm{~h}$ light/dark cycle at $25^{\circ} \mathrm{C}$, in accordance with the regulations of Soonchunhyang University Seoul Hospital.

\section{Statistical analysis}

Values are expressed as the mean \pm SEM and were evaluated by Student's t-test, or one- or two-way analysis of variance to compute the significance of differences between groups. Dunnett's test was used for comparisons of three or more groups. Data were considered significantly different at $p<0.05$.

\section{RESULTS}

\section{Patients with CKD show an increased level of HK2, and CKD mice-derived MSCs show impaired mitochondrial functionality}

To analyze the relationship between HK2 expression and CKD, we measured HK2 expression levels in the serum of patients with CKD. ELISA revealed a high level of HK2 in the serum of CKD patients compared with that of the healthy control group (Fig. 1A). To confirm that HK2 expression leads to metabolic dysregulation, we examined the serum concentration of MG, a cytotoxic glycolytic intermediate. MG levels were also significantly elevated in CKD patients compared with those in the healthy control group (Fig. 1B). These results are consistent with previous reports suggesting that HK2 overexpression may play a significant role in kidney injury (Smith et al., 2014; Lan et al., 2016).

To examine the relationship between HK2 and MSCs in the context of CKD, MSCs were isolated from both healthy and CKD mice. Immunoblotting revealed that CKD mice-derived MSCs exhibited elevated HK2 levels compared with those of their healthy counterparts, whereas HK1 expression remained unaffected (Fig. 1C). In addition, CKD-mMSCs had increased levels of mitochondrial reactive oxygen species (mtROS) and significantly decreased mitochondrial membrane potential, as assessed using the MitoSOX assay and TMRE staining, respectively (Fig. 1D, 1E). Taken together, these results indicate that in patients with CKD, increased HK2 expression promotes toxic glycolytic intermediates such as MG, and the effect of these intermediates might carry over to MSCs, undermining the functionality of mitochondria in harvested MSCs and their therapeutic potential.

\section{Melatonin improves oxidative stress and mitochondrial membrane potential by lowering mitochondrial HK2 expression in CKD-mMSCs}

Our data verifying the high HK2 levels in patients with CKD as well as in those with CKD-mMSCs suggested that CKDmMSCs may suffer from impaired mitochondrial function related to the role of HK2 in metabolism. Hence, we hypothesized that decreasing the expression of HK2 may increase the 

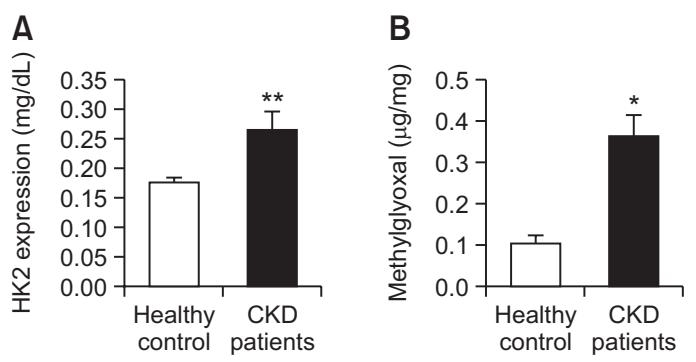

D
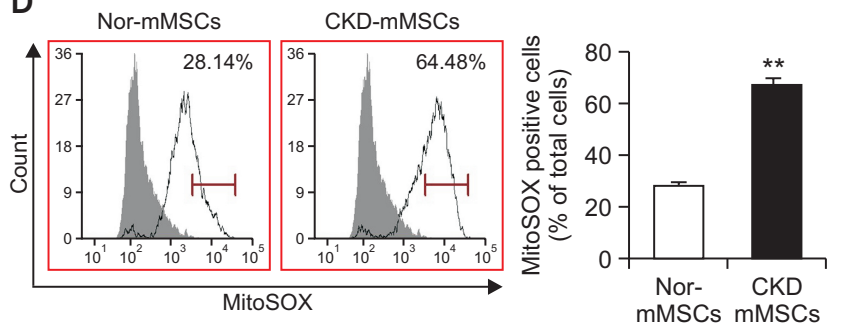
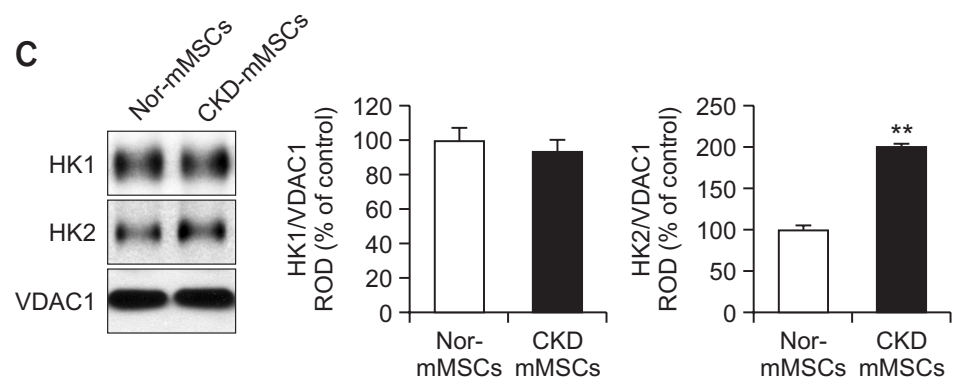

E
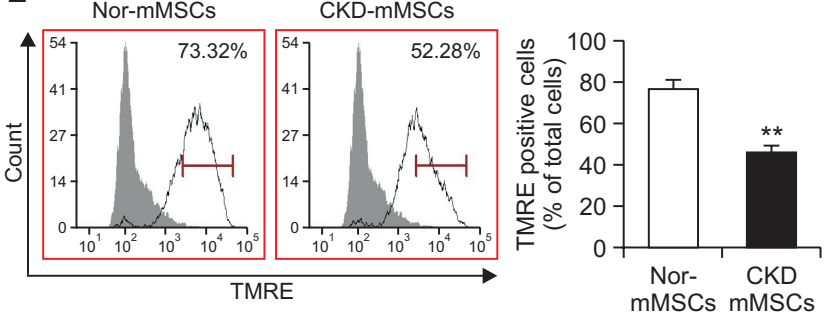

Fig. 1. Patients with chronic kidney disease (CKD) show an increased level of HK2, and CKD mice-derived mesenchymal stem/stromal cells (MSCs) show impaired mitochondrial functionality. (A) Serum samples from patients with CKD $(n=30)$ and healthy controls ( $n=30)$ were subjected to enzyme-linked immunosorbent assay (ELISA) for HK2. (B) Methylglyoxal (MG) content in human serum samples from the same experimental groups (CKD patients vs. healthy control group). (C) Expression of HK1 and HK2 in mitochondrial fractions of MSCs derived from the CKD mouse model (CKD-mMSCs) and normal control mice (Nor-mMSCs), as detected by immunoblotting ( $n=5)$. Expression levels of HK1 and HK2 were normalized relative to levels of the mitochondrial marker VDAC1. (D) Normal-mMSCs and CKD-mMSCs were assayed using MitoSOX to assess mitochondrial oxidative stress. MitoSOX signals were quantified using flow cytometry $(n=5)$. ( $E)$ NormalmMSCs and CKD-mMSCs were assayed using TMRE to assess the mitochondrial membrane potential. TMRE signals were quantified using flow cytometry $(n=5)$. Values represent the mean \pm standard error of the mean $(\mathrm{SEM}) .{ }^{*} p<0.05$ or ${ }^{* *} p<0.01$ vs. control.

functionality of MSCs by positively influencing mitochondrial activity. On a different note, we also questioned whether melatonin can modulate glycolytic overload via HK2. We previously reported that melatonin treatment provides protective effects against CKD, and improves mitochondrial functions in renal proximal tubular cells (Han et al., 2020) and CKD-MSCs (Yoon et al., 2020c). Others have proposed that melatonin can act as a glycolytic agent, which inhibits Warburg effect-dependent cancer cells by shifting the metabolic focus from anaerobic glycolysis to normal mitochondrial oxidative phosphorylation (Reiter et al., 2020). In light of these previous findings, we expected melatonin to downregulate the overabundant HK2 and its glycolytic side product MG in CKD-MSCs. Immunoblotting against HK2 showed increased HK2 expression levels in CKD-mMSCs, which was effectively reduced with melatonin treatment (Fig. 2A). Evaluation of mitochondrial oxidative stress and membrane potential demonstrated reduced mtROS and increased active mitochondria in melatonin-treated CKDmMSCs compared with those of the no-treatment group (Fig. $2 \mathrm{~B}, 2 \mathrm{C}$ ). We also determined the effect of melatonin on the glycolytic process in $\mathrm{TH} 1$ cells, which are human renal proximal tubule epithelial cells. Treatment of TH1 cells with $\mathrm{p}$-cresol increased the expression of HK2. However, melatonin treatment of $\mathrm{p}$-cresol-treated $\mathrm{TH} 1$ cells reduced the expression of HK2, similar to the results in CKD-mMSCs (Supplementary Fig. 1). Treatment of $\mathrm{TH} 1$ cells with $\mathrm{p}$-cresol also increased HK1 expression levels, which was reversed by treatment with luzindole, a melatonin inhibitor, confirming that the observed results were indeed dependent on melatonin. Taken together, these results indicate that melatonin can modulate HK2 expression to improve the functionality of mitochondria in CKD-
mMSCs.

\section{Melatonin decreases the production of HK2-related toxic metabolites and improves the metabolic activity of CKD- mMSCs}

After confirming the melatonin-mediated reduction of HK2 expression in CKD-mMSCs, we questioned whether the functional improvements observed with mitochondria in melatonin-treated CKD-mMSCs were related to the role of HK2 in glycolytic processes. Consistent with the differences in HK2 expression levels, the MG concentrations were elevated in CKD-mMSCs compared with those of mMSCs harvested from healthy mice (Nor-mMSCs), and this abnormal elevation of MG concentrations was inhibited by melatonin treatment (Fig. $3 A)$.

To determine whether this change in MG concentration is reflective of the larger shift in the bioenergetic profile of MSCs in response to melatonin treatment, we employed a seahorse assay to measure the OCR and ECAR. Plotting the OCR vs. ECAR for CKD-mMSCs, melatonin-treated CKD-mMSCs, and Nor-mMSCs (Fig. 3B) showed a consistently positive correlation with similar slopes, which suggests a similar metabolic profile across different conditions, and that the increase in OCR and ECAR values corresponded to an increase in the metabolic activity of mMSCs. Melatonin-treated CKD-mMSCs showed improved metabolic activity compared with that of the Nor-mMSC group, whereas CKD-mMSCs, which have no efficacy, and melatonin inhibitors were grouped together given their low metabolic activity. Specific measurements of ECAR (Fig. 3C) and OCR (Fig. 3D) demonstrated improved glycolysis and mitochondrial respiration, respectively. Overall, 
A
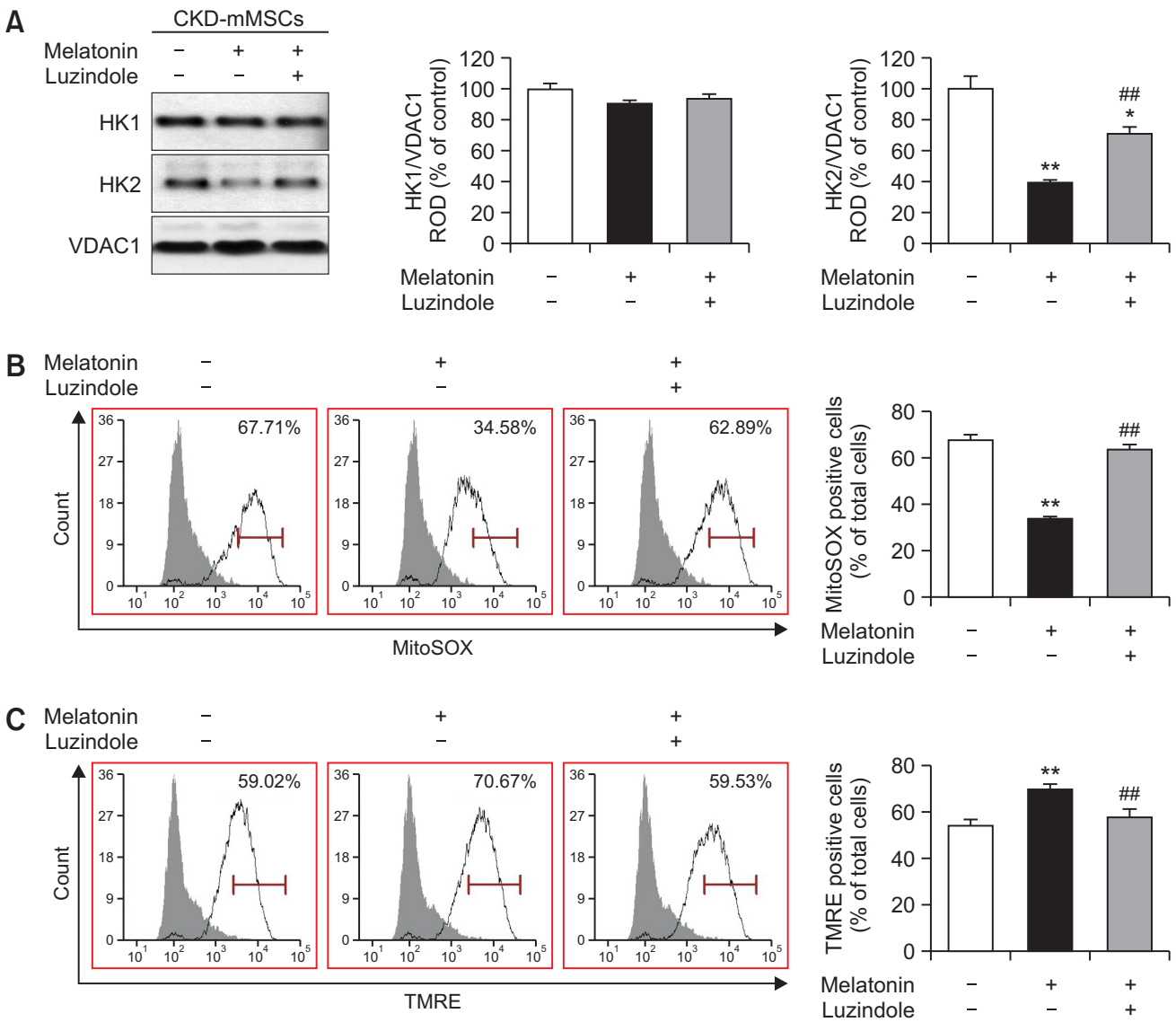

Fig. 2. Melatonin improved oxidative stress and mitochondrial membrane potential by lowering mitochondrial HK2 expression in chronic kidney disease mouse model-derived mesenchymal stem cells (CKD-mMSCs). (A) Immunoblotting results showing the expression of HK1 and HK2 in each experimental mMSC group: untreated CKD-mMSCs, CKD-mMSCs treated with melatonin, and melatonin-treated CKDmMSCs pre-treated with luzindole. Expression levels of HK1 and HK2 were normalized relative to VDAC1 levels. Values represent the mean \pm SEM $(n=3)$. ${ }^{*} p<0.05$ or ${ }^{* *} p<0.01$ vs. control; ${ }^{*} p<0.01$ vs. melatonin-treated CKD-mMSCs. (B) CKD-mMSCs from each experimental group assayed using MitoSOX to assess mitochondrial oxidative stress. MitoSOX signals were quantified using flow cytometry. Values represent the mean \pm SEM $(n=5)$. ${ }^{* *} p<0.01$ vs. control, ${ }^{*} p<0.01$ vs. melatonin-treated CKD-mMSCs. (C) CKD-mMSCs from each experimental group assayed using TMRE to assess mitochondrial membrane potential. TMRE signals were quantified using flow cytometry. Values represent the mean \pm SEM $(n=5-6)$. ${ }^{* *} p<0.01$ vs. control, ${ }^{\#} p<0.01$ vs. melatonin-treated CKD-mMSCs.

experimental mMSC groups with high MG levels were metabolically less active with worse performance in glycolysis and mitochondrial respiration, and melatonin treatment was able to enhance the metabolic performance of CKD-mMSCs closer to that of Nor-mMSCs through regulation of MG by influencing HK2 expression.

\section{Melatonin restores the cell cycle and expression patterns of cell cycle-associated proteins in CKD-mMSCs}

Since melatonin was found to be capable of regulating HK2 expression and MG concentration in CKD-mMSCs to restore their mitochondrial function and metabolic activity, we next analyzed whether these improvements translated into an enhanced proliferative capacity of MSCs, which is an important feature for autologous MSC treatment. To assess the improvement in cell proliferation induced by melatonin in CKDmMSCs, we determined the cell cycle of CKD-mMSCs using flow cytometry. CKD-mMSCs showed a decrease in the cell population in the S phase compared with that of Nor-mMSCs. However, melatonin treatment effectively restored the cell population in the S phase (Fig. 4A). We further determined the expression of cell cycle-associated proteins in CKD-mMSCs. Western blotting data showed marked downregulation in the expression of CDK2, cyclin E, CDK4, and cyclin D1, which are all important for the G1-phase to S-phase transition, whereas melatonin treatment successfully restored the expression of these proteins in CKD-mMSCs (Fig. 4B). Densitometric analysis showed that pre-treatment with luzindole almost completely negated the protective effect of melatonin, indicating that all improvements observed with CKD-mMSCs were attributable to the melatonin treatment. These results suggest that the ability of melatonin to regulate HK2 and its downstream metabolite MG may have implications for restoring cell proliferation in CKD-mMSCs.

\section{Melatonin improves the therapeutic efficacy of CKD- mMSCs in the CKD mouse model}

After confirming that melatonin treatment improved the mitochondrial function and cell proliferation of CKD-mMSCs, we tested whether melatonin could improve the therapeutic 
A
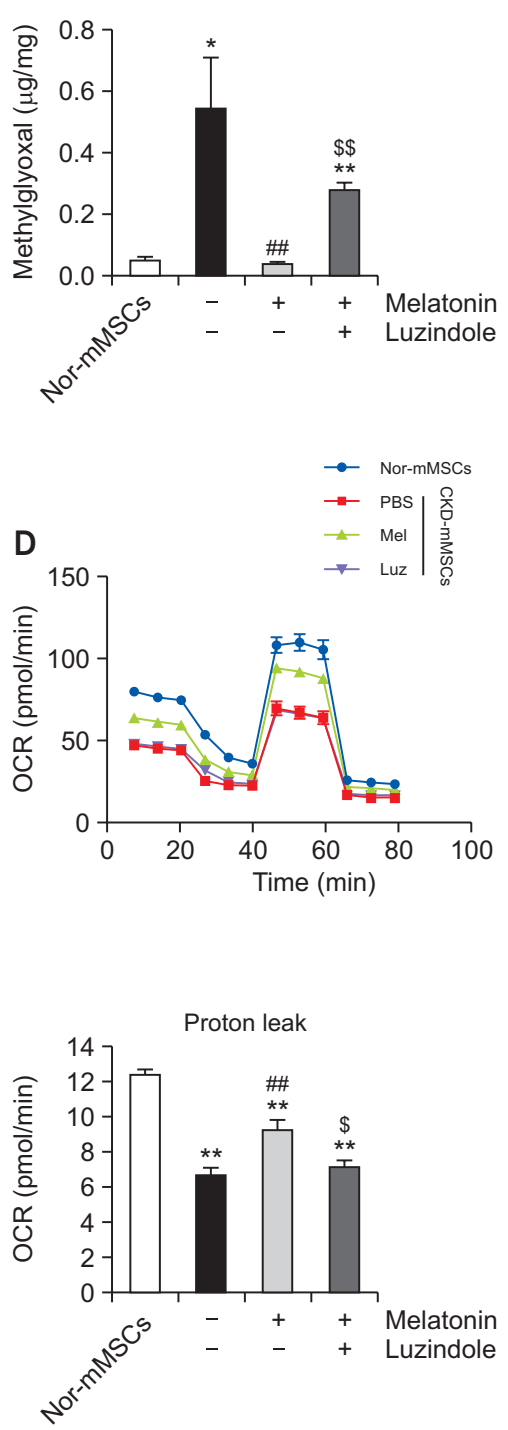
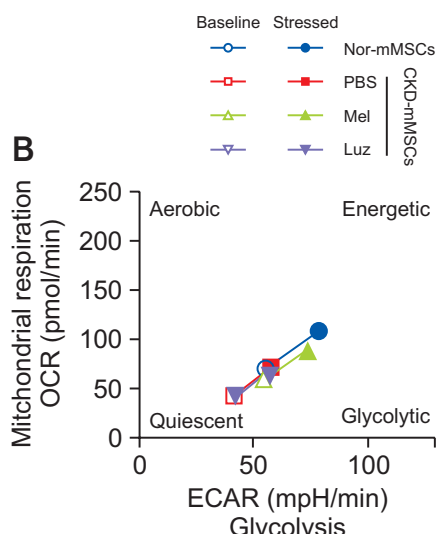

Glycolysis
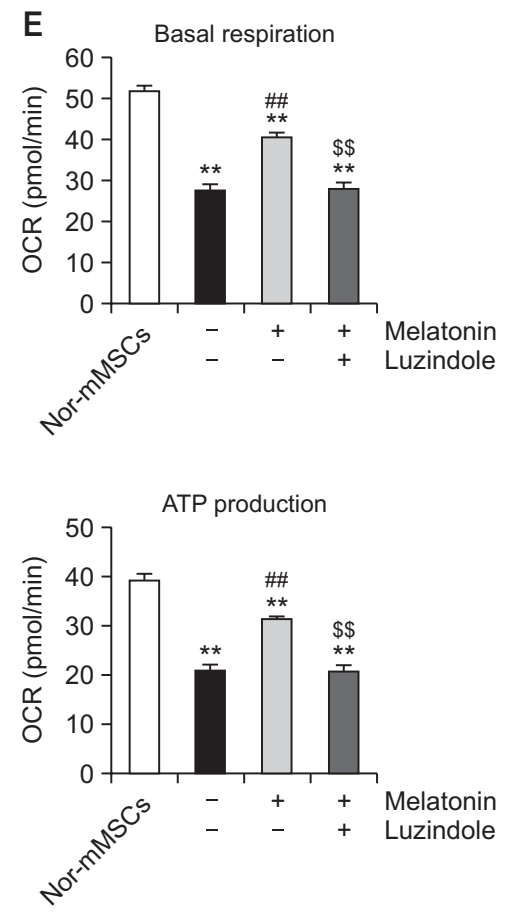
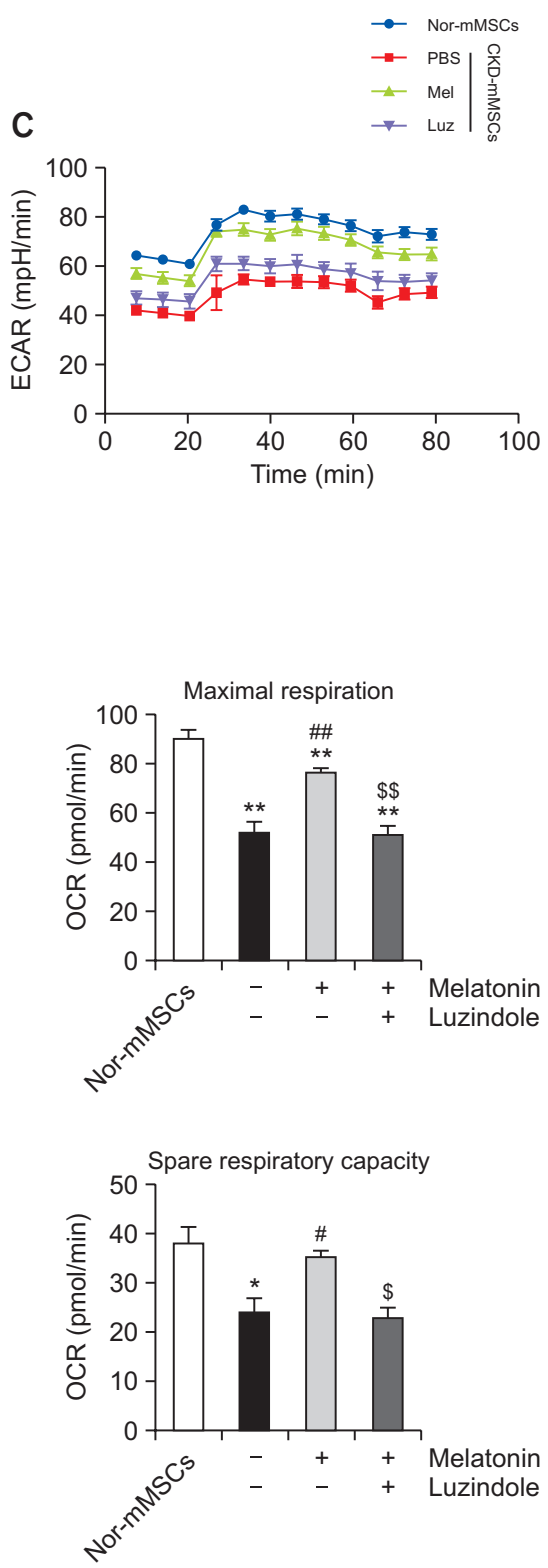

Fig. 3. Melatonin decreases the levels of HK2-related toxic metabolites and improves the metabolic activity of chronic kidney disease mouse model-derived mesenchymal stem cells (CKD-mMSCs). (A) Measurement of methylglyoxal (MG) in mMSCs derived from normal healthy mice (Nor-mMSCs) or CKD mice (CKD-mMSCs) with enzyme-linked immunosorbent assay (ELISA). CKD-mMSCs were either treated with melatonin alone or pre-treated with luzindole before melatonin treatment. Values represent the mean $\pm \mathrm{SEM}(\mathrm{n}=5)$. ${ }^{*} p<0.05$ or ${ }^{* *} p<0.01$ vs. Nor-mMSCs; ${ }^{\#} p<0.01$ vs. CKD-mMSCs; ${ }^{\$ \$} p<0.01$ vs. melatonin-treated CKD-mMSCs. (B) Mitochondrial oxygen consumption rate (OCR) vs. extracellular acidification rate (ECAR) equated to the glycolytic rate of Nor-mMSCs, CKD-mMSCs, and CKD-mMSCs with melatonin treatment (Mel), or melatonin-treated CKD-mMSCs pre-treated with luzindole (Luz). Assessment of the baseline metabolic profile of each mMSC group was followed by a stress test to measure the metabolic shift toward the energetic state. (C) Seahorse assay for determining the ECAR of Nor-mMSCs and CKD-mMSCs under different experimental conditions. (D) Mitochondrial OCR of each group over time (min). (E) Histogram representing the bioenergetics parameters are based on the OCR data by administering oligomycin $(20 \mathrm{~min}, 1 \mu \mathrm{M})$, FCCP $(40 \mathrm{~min}, 0.75 \mu \mathrm{M})$, and antimycin A $(60 \mathrm{~min}, 1 \mu \mathrm{M})$. Values represent the mean \pm SEM $(\mathrm{n}=5) .{ }^{*} p<0.05$ or ${ }^{* *} p<0.01 \mathrm{vs}$. Nor-mMSCs; ${ }^{\#} p<0.05$ or ${ }^{\# \#} p<0.01$ vs. CKD-mMSCs; ${ }^{\$} p<0.05$ or ${ }^{\$ \$} p<0.01$ vs. melatonin-treated CKD-mMSCs.

efficacy of CKD-mMSCs in the CKD mouse model. We examined the renal function recovery in CKD mice after the injection of melatonin-treated CKD-mMSCs by measuring serum blood urea nitrogen (BUN) and creatinine levels. When Nor-mMSCs were injected into CKD mice, BUN and creati- nine levels decreased, whereas the injection of CKD-mMSCs did not decrease BUN and creatinine levels. However, when melatonin-treated CKD-mMSCs were injected into CKD mice, BUN and creatinine levels significantly increased (Fig. 5A, $5 B)$. Further, treatment with luzindole reversed these effects, 
A

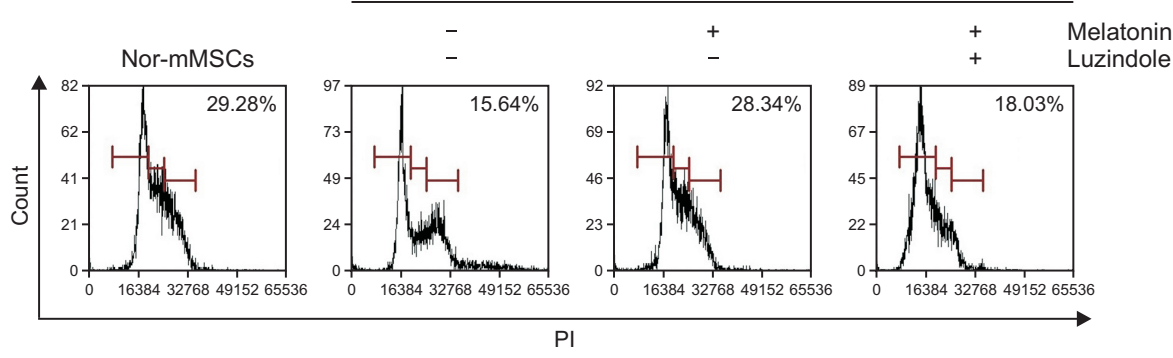

B

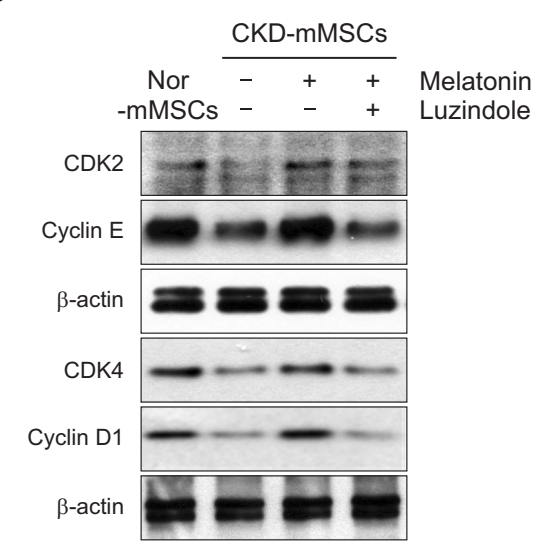

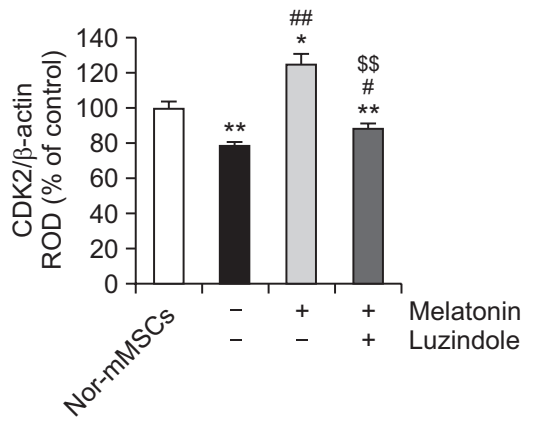
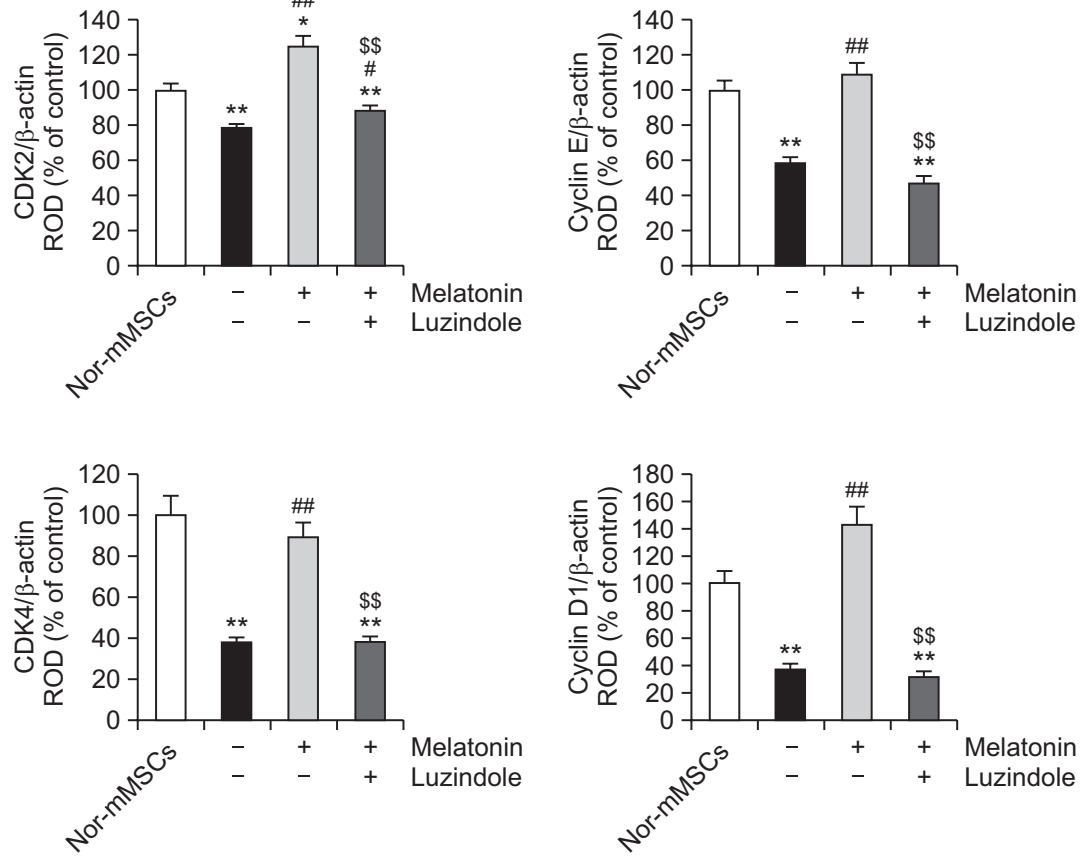
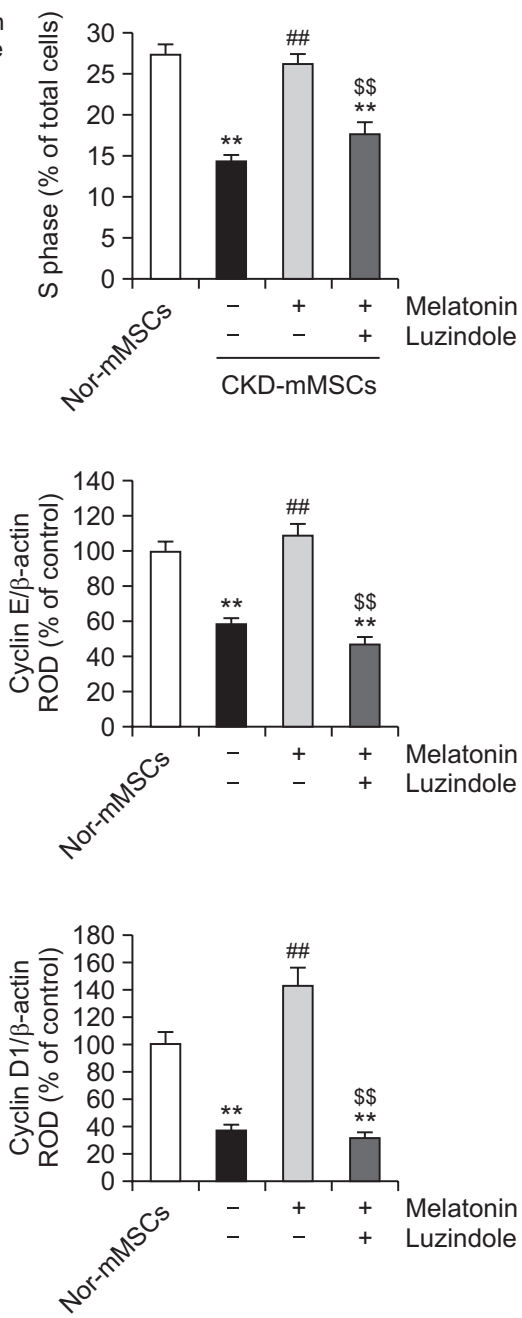

Fig. 4. Melatonin restores the cell cycle and expression patterns of cell cycle-associated proteins in chronic kidney disease mouse-derived mesenchymal stem cells (CKD-mMSCs). (A) Cell cycle analysis of control mouse MSCs (Nor-mMSCs) and CKD-mMSCs using flow cytometry. (B) Immunoblotting data showing the expression of cell cycle-associated proteins, CDK2, cyclin E, CDK4, and cyclin D1, in NormMSCs, CKD-mMSCs, CKD-mMSCs with melatonin treatment, or melatonin-treated CKD-mMSCs pre-treated with luzindole. Band signals from western blotting were quantified by densitometry and were normalized to $\beta$-actin levels $(n=3)$. Values represent the mean \pm SEM. ${ }^{*} p<0.05$ or ${ }^{* *} p<0.01$ vs. Nor-mMSCs; ${ }^{\#} p<0.05$ or ${ }^{\# \#} p<0.01$ vs. CKD-mMSCs; ${ }^{\$ \$} p<0.01$ vs. melatonin treated CKD-mMSCs.

confirming that the observed effects were due to melatonin treatment (Fig. 5A, 5B). These results suggest that melatonin enhances the mitochondrial function and cell proliferation of CKD-mMSCs, and improves the therapeutic efficacy of CKDmMSCs in a CKD mouse model.

\section{DISCUSSION}

Sustained damage to the kidney tissues leads to CKD and progressive loss of kidney function. The compromised glomerular filtration, excretion, and reabsorption causes the increased circulation of uremic toxins. These effects can be detrimental to the viability and normal functions of MSCs and limit their application in regenerative therapy for CKD patients. Although previous studies to improve the efficacy of MSC- based therapy for CKD have demonstrated that melatonin treatment provides a broad range of protective effects (Han et al., 2019; Hu and Li, 2019; Yoon et al., 2020c), the complex biological mechanisms underlying this phenomenon have not been well elucidated. In this study, we demonstrated that treatment of MSCs with melatonin modulates the glycolytic process in these cells by influencing HK2 expression and MG concentration, which improved mitochondrial function and cell proliferation in CKD-mMSCs (Fig. 6).

Although recent studies on the mechanisms underlying the therapeutic effect of melatonin against kidney diseases have focused on its effect on mitochondrial function (Yoon et al., 2020a), homeostasis (Qi and Wang, 2020), and autophagy (Yoon et al., 2019), whether melatonin modulates the glycolytic pathways in CKD remains a relatively under-researched topic. Several review articles in the field of cancer research 
A

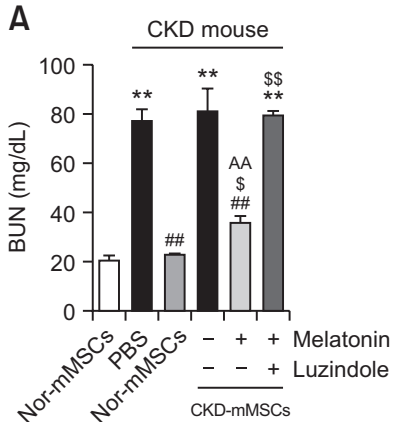

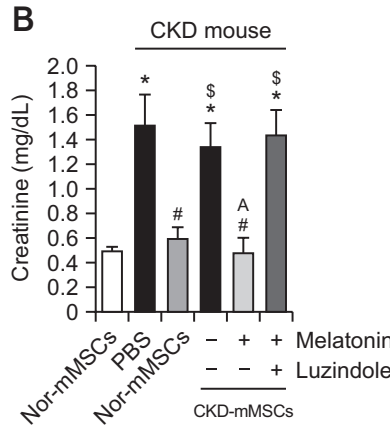

Fig. 5. Melatonin treatment improves the therapeutic efficacy of chronic kidney disease mouse-derived mesenchymal stem cells (CKD-mMSCs). Measurement of blood urea nitrogen (A) and creatinine $(B)$ levels in the serum of CKD mice. Each CKD mouse was injected with phosphate-buffered saline (PBS) or with control mouse MSCs (Nor-mMSCs), CKD-mMSCs, melatonin-treated CKD-mMSCs, or melatonin-treated CKD-mMSCs pretreated with luzindole. Values represent the mean \pm SEM. ${ }^{*} p<0.05$ or ${ }^{* *} p<0.01$ vs. healthy mouse; ${ }^{\#} p<0.05$ or ${ }^{\#} p<0.01$ vs. PBS; ${ }^{\$} p<0.05$ or ${ }^{\$ \$} p<0.01$ vs. Nor-mMSCs; ${ }^{A} p<0.05,{ }^{A A} p<0.01$ vs. CKD-mMSCs. have elucidated the metabolism-altering anti-cancer effects of melatonin (Li et al., 2017; Reiter et al., 2017; Talib, 2018; Wang et al., 2018). For example, melatonin has been found to inhibit tumor growth in Ewing sarcoma (Sanchez-Sanchez et al., 2015), leiomyosarcoma (Mao et al., 2016), breast cancer (Hill et al., 2015), prostate cancer (Hevia et al., 2017), and hepatocellular carcinoma (Feng et al., 2020) by suppressing excessive aerobic glycolysis (Warburg effect). Furthermore, a recent review article suggested that the modulation of glycolytic functions by melatonin is also associated with inhibition of various diseases (Reiter et al., 2021). On a different note, HK2-mediated glycolytic dysregulation has been implicated in fibrotic processes (Xie et al., 2015; Yin et al., 2019; Gopu et al., 2020), which is an important part of the CKD pathophysiology. With the above findings providing a hint for the link between melatonin and glycolysis in CKD, our present study provides more tangible evidence for the melatonin-mediated modulation of glycolysis in the CKD environment, although our experiments were more focused on MSCs.

hMSCs are among the most commonly used adult stem cells in experimental cell therapies (Pittenger et al., 2019). Despite the initial focus on the multi-potency of MSCs as the rationale behind applying MSC-based cell therapy, more recent studies have led to the novel perspective that the more significant aspect of MSCs for clinical applications comes from

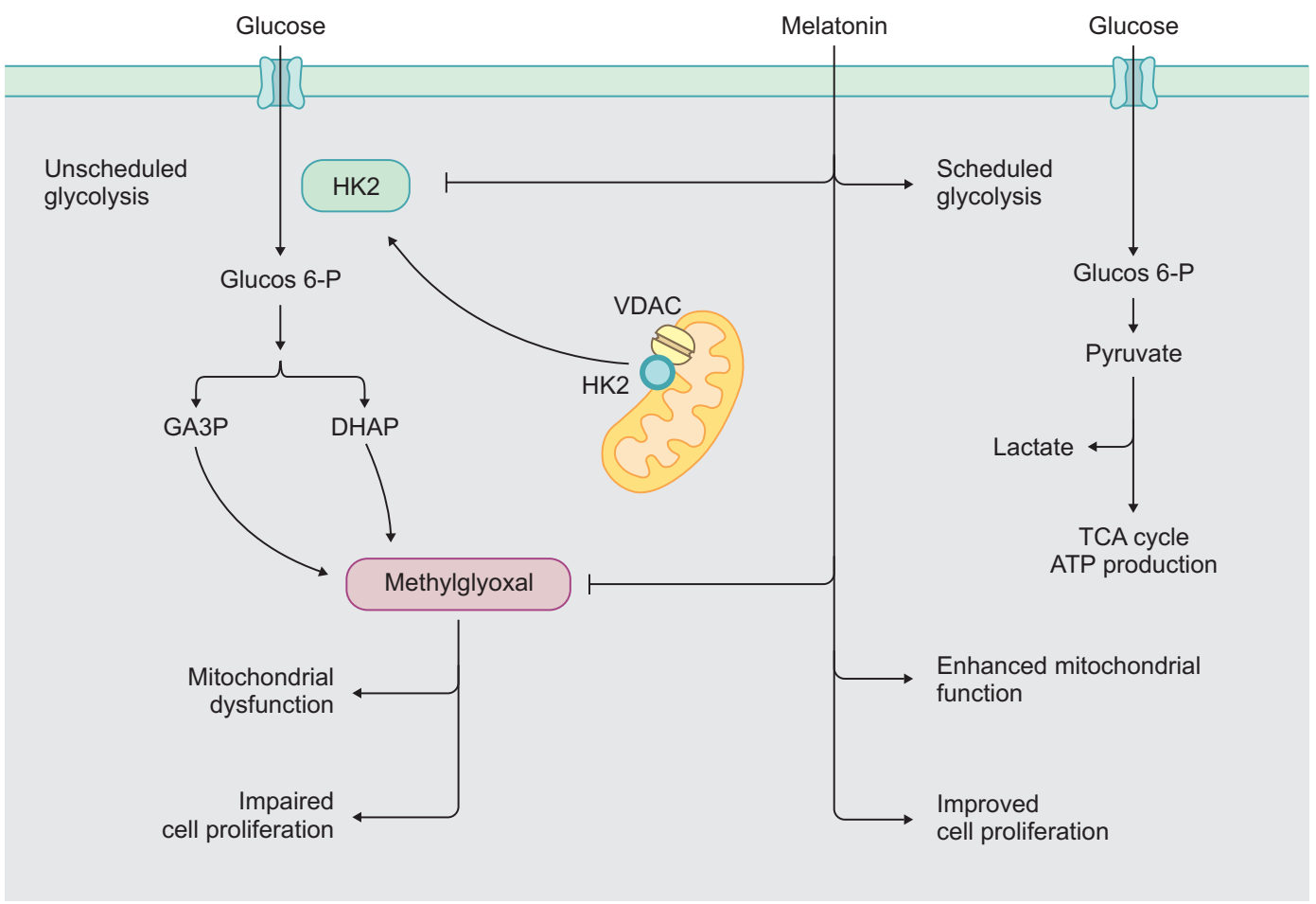

Fig. 6. Schematic representation of the proposed protective effect of melatonin treatment for mouse mesenchymal stem/stromal cells in chronic kidney disease (CKD-mMSCs) through modulating hexokinase-2 (HK2), thereby reducing the damage caused by the accumulation of methylglyoxal (MG) during abnormal or unscheduled glycolysis. In CKD-mMSCs, HK2 dissociates from the mitochondrial voltage-dependent anion channel (VDAC) and induces excessive production of a toxic glycolytic intermediate product (MG). This leads to mitochondrial dysfunction and impaired cell proliferation. Melatonin treatment of CKD-mMSCs enhances mitochondrial functions and rescues the normal expression of cell cycle-related proteins. 
their ability to secrete immunoregulatory and pro-regenerative factors (Yuan et al., 2019). There is growing interest in using allogeneic and culture-expanded cells for MSC-based cell therapy because their characteristics are well documented and they have potential for mass production. Nonetheless, this approach also lends itself to other barriers for the therapeutic application of MSCs, as the specific morbidities of MSC donors or extensive cell culturing can alter the metabolism of MSCs and compromise their therapeutic potential (Mastrolia et al., 2019). Therefore, understanding the underlying regulatory mechanisms of MSC metabolism and functions, and finding ways to control or bioengineer these properties of MSCs may help to drastically improve the efficacy of MSC-based therapy for specific target diseases. In this light, our successful attempt to improve the glycolytic profile of CKD-MSCs with melatonin treatment may help build the foundation for additional research efforts to develop an effective cure for CKD.

Although melatonin is primarily known for its role in sleep regulation, the broad impact of melatonin on regenerative processes in various tissues provides an excellent entry point for exploring different regulatory mechanisms for therapeutic purposes (Tordjman et al., 2017). For example, our previous work with CKD-mMSCs revealed that melatonin enhances the viability, motility, stemness, and proliferation of MSCs (Han et al., 2019; Lee et al., 2020). We also reported that melatonintreated MSCs can better withstand the pathophysiological conditions of the CKD environment when used for CKD cell therapy in a mouse model (Han et al., 2019; Lee et al., 2019). In addition, we established specific mechanisms for the protective effects of melatonin against CKD in MSCs involving antioxidant enzymes (Lee et al., 2020), proangiogenic cytokines (Lee et al., 2020), and cellular prion proteins (Han et al., 2019). Our current research revealed that the glycolytic enzyme HK2 and its intermediate metabolite MG can be a target of modulation in melatonin treatment for CKD, providing another potential option for treating CKD-MSCs prior to their use in further cell-based CKD therapies.

In this study, we demonstrated that melatonin restores mitochondrial function and promotes cell proliferation by inhibiting the expression of HK2 and the accumulation of MG. There are conflicting reports on the correlation between mitochondrial function and cell proliferation. In cancer cells with active cell division, mitochondrial function is decreased and glycolysis is increased (Galli et al., 2003; Antico Arciuch et al., 2012). Recent studies have shown that mitochondrial function restoration can promote cell proliferation (Yan et al., 2017; Yao et al., 2019). Therefore, in this study, melatonin may have promoted cell proliferation by enhancing mitochondrial function. Moreover, there is a possibility that melatonin directly promotes cell proliferation through other mechanisms.

Given that the clinical treatment of CKD patients with hMSC-based cell therapy requires substantial improvements in efficacy, we examined the efficacy of the melatonin-mediated inhibition of HK2 expression in CKD-mMSCs derived from a CKD mouse model. Not only were abnormal elevations of HK2 and its downstream metabolite MG inhibited by melatonin treatment, but also mitochondrial function as assessed by the respiratory OCR, and cell proliferation as measured by the expression of pro-proliferative proteins, were also improved. This experimental outcome may reflect the significant role of HK2 in the cellular activity and metabolism of MSCs. It is noteworthy that the broad impact of melatonin on CKD-
mMSCs influences other metabolic processes in addition to hexokinase-induced glycolysis. Therefore, identification and characterization of the various intertwined metabolic regulators under the influence of melatonin with respect to MSCs in a CKD condition may build a foundation for developing more effective therapeutic strategies, cell-based or otherwise, for treating CKD and other kidney disorders.

\section{CONFLICT OF INTEREST}

The authors have no conflict of interest to declare.

\section{ACKNOWLEDGMENTS}

This research was supported by the Bio \& Medical Technology Development Program of the National Research Foundation (NRF) funded by the Korean government (MSIT) (2019M3A9H1103495).

\section{REFERENCES}

Antico Arciuch, V. G., Elguero, M. E., Poderoso, J. J. and Carreras, M. C. (2012) Mitochondrial regulation of cell cycle and proliferation. Antioxid. Redox Signal. 16, 1150-1180.

Bhatia, D., Chung, K. P., Nakahira, K., Patino, E., Rice, M. C., Torres, L. K., Muthukumar, T., Choi, A. M., Akchurin, O. M. and Choi, M. E. (2019) Mitophagy-dependent macrophage reprogramming protects against kidney fibrosis. JCI Insight 4, e132826.

Breyer, M. D. and Susztak, K. (2016) Developing treatments for chronic kidney disease in the 21st century. Semin. Nephrol. 36, 436-447.

Feng, J., Li, J., Wu, L., Yu, Q., Ji, J., Wu, J., Dai, W. and Guo, C. (2020) Emerging roles and the regulation of aerobic glycolysis in hepatocellular carcinoma. J. Exp. Clin. Cancer Res. 39, 126.

Fu, Z., Jiao, Y., Wang, J., Zhang, Y., Shen, M., Reiter, R. J., Xi, Q. and Chen, Y. (2020) Cardioprotective role of melatonin in acute myocardial infarction. Front. Physiol. 11, 366.

Galli, S., Labato, M. I., Bal de Kier Joffé, E., Carreras, M. C. and Poderoso, J. J. (2003) Decreased mitochondrial nitric oxide synthase activity and hydrogen peroxide relate persistent tumoral proliferation to embryonic behavior. Cancer Res. 63, 6370-6377.

GBD Chronic Kidney Disease Collaboration (2020) Global, regional, and national burden of chronic kidney disease, 1990-2017: a systematic analysis for the Global Burden of Disease Study 2017. Lancet 395, 709-733.

Gopu, V., Fan, L., Shetty, R. S., Nagaraja, M. R. and Shetty, S. (2020) Caveolin-1 scaffolding domain peptide regulates glucose metabolism in lung fibrosis. JCl Insight 5, e137969.

Han, Y. S., Kim, S. M., Lee, J. H., Jung, S. K., Noh, H. and Lee, S. H. (2019) Melatonin protects chronic kidney disease mesenchymal stem cells against senescence via PrPC-dependent enhancement of the mitochondrial function. J. Pineal Res. 66, e12535.

Han, Y. S., Yoon, Y. M., Go, G., Lee, J. H. and Lee, S. H. (2020) Melatonin protects human renal proximal tubule epithelial cells against high glucose-mediated fibrosis via the cellular prion protein-TGFbeta-Smad signaling axis. Int. J. Med. Sci. 17, 1235-1245.

Hevia, D., Gonzalez-Menendez, P., Fernandez-Fernandez, M., Cueto, S., Rodriguez-Gonzalez, P., Garcia-Alonso, J. I., Mayo, J. C. and Sainz, R. M. (2017) Melatonin decreases glucose metabolism in prostate cancer cells: a (13)C stable isotope-resolved metabolomic study. Int. J. Mol. Sci. 18, 1620.

Hickson, L. J., Eirin, A. and Lerman, L. O. (2016) Challenges and opportunities for stem cell therapy in patients with chronic kidney disease. Kidney Int. 89, 767-778.

Hill, S. M., Belancio, V. P., Dauchy, R. T., Xiang, S., Brimer, S., Mao, L., Hauch, A., Lundberg, P. W., Summers, W., Yuan, L., Frasch, T. and 
Blask, D. E. (2015) Melatonin: an inhibitor of breast cancer. Endocr. Relat. Cancer 22, R183-R204.

$\mathrm{Hu}, \mathrm{C}$. and Li, L. (2018) Preconditioning influences mesenchymal stem cell properties in vitro and in vivo. J. Cell. Mol. Med. 22, 1428-1442.

$\mathrm{Hu}, \mathrm{C}$. and Li, L. (2019) Melatonin plays critical role in mesenchymal stem cell-based regenerative medicine in vitro and in vivo. Stem Cell Res. Ther. 10, 13

Kato, M. and Natarajan, R. (2019) Epigenetics and epigenomics in diabetic kidney disease and metabolic memory. Nat. Rev. Nephrol. 15, 327-345.

Keshtkar, S., Azarpira, N. and Ghahremani, M. H. (2018) Mesenchymal stem cell-derived extracellular vesicles: novel frontiers in regenerative medicine. Stem Cell Res. Ther. 9, 63.

Lan, R., Geng, H., Singha, P. K., Saikumar, P., Bottinger, E. P., Weinberg, J. M. and Venkatachalam, M. A. (2016) Mitochondrial pathology and glycolytic shift during proximal tubule atrophy after ischemic AKI. J. Am. Soc. Nephrol. 27, 3356-3367.

Lee, J. H., Yoon, Y. M., Han, Y. S., Jung, S. K. and Lee, S. H. (2019) Melatonin protects mesenchymal stem cells from autophagy-mediated death under ischaemic ER-stress conditions by increasing prion protein expression. Cell Prolif. 52, e12545.

Lee, J. H., Yoon, Y. M., Song, K. H., Noh, H. and Lee, S. H. (2020) Melatonin suppresses senescence-derived mitochondrial dysfunction in mesenchymal stem cells via the HSPA1L-mitophagy pathway. Aging Cell 19, e13111.

Lee, J. H., Yun, C. W., Hur, J. and Lee, S. H. (2018) Fucoidan rescues p-cresol-induced cellular senescence in mesenchymal stem cells via FAK-Akt-TWIST axis. Mar. Drugs 16, 121.

Li, Y., Li, S., Zhou, Y., Meng, X., Zhang, J. J., Xu, D. P. and Li, H. B. (2017) Melatonin for the prevention and treatment of cancer. Oncotarget 8, 39896-39921.

Liu, H., Liu, N., Cheng, Y., Jin, W., Zhang, P., Wang, X., Yang, H., Xu, X., Wang, Z. and Tu, Y. (2017) Hexokinase 2 (HK2), the tumor promoter in glioma, is downregulated by miR-218/Bmi1 pathway. PLOS ONE 12, e0189353.

Lv, J. C. and Zhang, L. X. (2019) Prevalence and disease burden of chronic kidney disease. Adv. Exp. Med. Biol. 1165, 3-15.

Mao, L., Dauchy, R. T., Blask, D. E., Dauchy, E. M., Slakey, L. M., Brimer, S., Yuan, L., Xiang, S., Hauch, A., Smith, K., Frasch, T., Belancio, V. P., Wren, M. A. and Hill, S. M. (2016) Melatonin suppression of aerobic glycolysis (Warburg effect), survival signalling and metastasis in human leiomyosarcoma. J. Pineal Res. 60, 167-177.

Mastrolia, I., Foppiani, E. M., Murgia, A., Candini, O., Samarelli, A. V., Grisendi, G., Veronesi, E., Horwitz, E. M. and Dominici, M. (2019) Challenges in clinical development of mesenchymal stromal/stem cells: concise review. Stem Cells Transl. Med. 8, 1135-1148.

Nigam, S. K. and Bush, K. T. (2019) Uraemic syndrome of chronic kidney disease: altered remote sensing and signalling. Nat. Rev. Nephrol. 15, 301-316.

Ohashi, N., Ishigaki, S. and Isobe, S. (2019) The pivotal role of melatonin in ameliorating chronic kidney disease by suppression of the renin-angiotensin system in the kidney. Hypertens. Res. 42, 761768.

Pittenger, M. F., Discher, D. E., Péault, B. M., Phinney, D. G., Hare, J. M. and Caplan, A. I. (2019) Mesenchymal stem cell perspective: cell biology to clinical progress. NPJ Regen. Med. 4, 22.

Qi, X. and Wang, J. (2020) Melatonin improves mitochondrial biogenesis through the AMPK/PGC1alpha pathway to attenuate ischemia/ reperfusion-induced myocardial damage. Aging (Albany NY) 12 , 7299-7312.

Rabbani, N. and Thornalley, P. J. (2019) Hexokinase-2 glycolytic overload in diabetes and ischemia-reperfusion injury. Trends Endocrinol. Metab. 30, 419-431.

Reiter, R. J., Rosales-Corral, S. A., Tan, D. X., Acuna-Castroviejo, D., Qin, L., Yang, S. F. and Xu, K. (2017) Melatonin, a full service anti-cancer agent: Inhibition of initiation, progression and metastasis. Int. J. Mol. Sci. 18, 843.

Reiter, R. J., Sharma, R., Ma, Q., Rorsales-Corral, S. and de Almeida
Chuffa, L. G. (2020) Melatonin inhibits Warburg-dependent cancer by redirecting glucose oxidation to the mitochondria: a mechanistic hypothesis. Cell. Mol. Life Sci. 77, 2527-2542.

Reiter, R. J., Sharma, R. and Rosales-Corral, S. (2021) Anti-Warburg effect of melatonin: a proposed mechanism to explain its inhibition of multiple diseases. Int. J. Mol. Sci. 22, 764.

Romagnani, P., Remuzzi, G., Glassock, R., Levin, A., Jager, K. J., Tonelli, M., Massy, Z., Wanner, C. and Anders, H. J. (2017) Chronic kidney disease. Nat. Rev. Dis. Primers 3, 17088.

Sanchez-Sanchez, A. M., Antolin, I., Puente-Moncada, N., Suarez, S., Gomez-Lobo, M., Rodriguez, C. and Martin, V. (2015) Melatonin cytotoxicity is associated to Warburg effect inhibition in Ewing sarcoma cells. PLoS ONE 10, e0135420.

Smith, J. A., Stallons, L. J. and Schnellmann, R. G. (2014) Renal cortical hexokinase and pentose phosphate pathway activation through the EGFR/Akt signaling pathway in endotoxin-induced acute kidney injury. Am. J. Physiol. Renal Physiol. 307, F435-F444.

Talib, W. H. (2018) Melatonin and cancer hallmarks. Molecules 23, 518.

Thalakiriyawa, D. S., Jayasooriya, P. R. and Dissanayaka, W. L. (2021) Regenerative potential of mesenchymal stem cell derived extracellular vesicles. Curr. Mol. Med. doi: 10.2174/156652402166 6210211114453 [Online ahead of print]

Tordjman, S., Chokron, S., Delorme, R., Charrier, A., Bellissant, E., Jaafari, N. and Fougerou, C. (2017) Melatonin: Pharmacology, functions and therapeutic benefits. Curr. Neuropharmacol. 15, 434-443.

Wang, Y., Wang, P., Zheng, X. and Du, X. (2018) Therapeutic strategies of melatonin in cancer patients: a systematic review and metaanalysis. OncoTargets Ther. 11, 7895-7908.

Xie, N., Tan, Z., Banerjee, S., Cui, H., Ge, J., Liu, R. M., Bernard, K. Thannickal, V. J. and Liu, G. (2015) Glycolytic reprogramming in myofibroblast differentiation and lung fibrosis. Am. J. Respir. Crit. Care Med. 192, 1462-1474.

Yan, X. J., Yu, X., Wang, X. P., Jiang, J. F., Yuan, Z. Y., Lu, X., Lei, F. and Xing, D. M. (2017) Mitochondria play an important role in the cell proliferation suppressing activity of berberine. Sci. Rep. 7, 41712.

Yao, C. H., Wang, R., Wang, Y., Kung, C. P., Weber, J. D. and Patti, G. J. (2019) Mitochondrial fusion supports increased oxidative phosphorylation during cell proliferation. eLife 8, e41351.

Yin, X., Choudhury, M., Kang, J. H., Schaefbauer, K. J., Jung, M. Y. Andrianifahanana, M., Hernandez, D. M. and Leof, E. B. (2019) Hexokinase 2 couples glycolysis with the profibrotic actions of TGF- $\beta$. Sci. Signal. 12, eaax4067.

Yoon, Y. M., Go, G., Yun, C. W., Lim, J. H., Lee, J. H. and Lee, S. H. (2020a) Melatonin suppresses renal cortical fibrosis by inhibiting cytoskeleton reorganization and mitochondrial dysfunction through regulation of miR-4516. Int. J. Mol. Sci. 21, 5323.

Yoon, Y. M., Go, G., Yun, C. W., Lim, J. H. and Lee, S. H. (2020b) Knockdown of CK2alpha reduces $\mathrm{P}$-cresol-induced fibrosis in human renal proximal tubule epithelial cells via the downregulation of profilin-1. Int. J. Med. Sci. 17, 2850-2860.

Yoon, Y. M., Kim, H. J., Lee, J. H. and Lee, S. H. (2019) Melatonin enhances mitophagy by upregulating expression of heat shock 70 kDa protein $1 \mathrm{~L}$ in human mesenchymal stem cells under oxidative stress. Int. J. Mol. Sci. 20, 4545.

Yoon, Y. M., Lee, J. H., Song, K. H., Noh, H. and Lee, S. H. (2020c) Melatonin-stimulated exosomes enhance the regenerative potential of chronic kidney disease-derived mesenchymal stem/stromal cells via cellular prion proteins. J. Pineal Res. 68, e12632.

Yuan, X., Logan, T. M. and Ma, T. (2019) Metabolism in human mesenchymal stromal cells: a missing link between hMSC biomanufacturing and therapy? Front. Immunol. 10, 977.

Yun, C. W. and Lee, S. H. (2019) Potential and therapeutic efficacy of cell-based therapy using mesenchymal stem cells for acute/chronic kidney disease. Int. J. Mol. Sci. 20, 1619. 